\title{
MERCADO DE TRABAJO Y FUERZAS SOCIALES EN EUSKAL HERRIA SUR
}

\author{
Iñaki Martínez de Luna Pérez de Arriba \\ (Dirección de Estadistica. Gobierno Vasco)
}

\section{PRECISIONES METODOLOGICAS}

Cuando analizamos la evolución de la población activa hemos de tener en cuenta que a partir del segundo trimestre de 1980 los resultados que ofrece la EPA - principal fuente estadística a utilizar para estos menesteres- se refieren a quienes tienen más de 15 años, en contraste con el período anterior en que se recogía a partit de los 14 años inclusive.

Esto supone un descenso de la población activa, que dificulta la comparación en términos homogéneos entre ambos períodos.

Tratando de evitar en lo posible este inconveniente, presentamos en el cuadro 1 los datos homogéneos a partir de 1979, inclusive. Es decir, los datos desde el citado año son los referentes a la población económicamente activa de 16 o más años.

\section{ERROR MUESTRAL DE LA EPA}

Según un estudio realizado por Angeles Iztueta, Ia Encuesta de Pobla. ción Activa (EPA), no puede cumplir en la Comunidad Autónoma Vasca ni, aún menos, en cada una de las provincias que la componen (Álava, Vizcaya y Guipúzcoa), los objetivos que pretende aicanzar a nivel estatal, principalmente por dos motivos: 1) Las muestras resultan pequeñas en las provincias aun para analizat características globales. 2) La estratificación 
no es adecuada para el ámbito de Euskal Herria Sur, ya que, diseñada en un marco estatal, no asegura la representación en la muestra de las poblaciones importantes de nuestro país.

Concretamente, a nivel de Alava, Guipúzcoa y Vizcaya, cada una por separado, sólo podemos hablar de grandes colectivos (activos, inactivos y ocupados en sentido estricto) debido a los etrotes de muestreo existentes.

Un colectivo tan importante como es el de los parados es estimado en su volumen con un error del $\pm 20,5 \%$ en el caso de Álava; del $\pm 12,6 \%$ en Guipúzcoa y del $16,6 \%$ para Vizcaya. Todo ello para un nivel de confranza del $95 \%$ en la utilización de datos puntuales.

Una vez visto el error de muestreo que se da para ciertos colectivos, puede fácilmente imaginarse que este problema se agudizatá en el caso de entrar en datos desagregados, alcanzando cotas que invalidan su utilización puntual. Tal es el caso de la desagregación por edades.

\section{a) Series con datos de la EPA}

Para una utilización de series, el problema queda así:

1) Variaciones trimestrales; no son significativas, ya que no hay evidencia estadística para afirmar que los pequeños saltos no se deban a los errores de muestreo.

2) Variaciones anuales: son significativas; no pueden ser explicadas únicamente en base a los exrotes de muestreo.

Por todo ello, siempre que nos sea posible evitaremos en este trabajo la utilización de datos puntuales, dando paso al uso de series basadas en medias anuales, siempre que se trate de datos sacados de la Encuesta de Población Activa.

\section{Comunidad Autónoma Vasca y Navarra}

El grave contencioso suscitado en la superestructura política vasca, entre fuerzas nacionalistas y estatalistas, sobre la integración o no de Navarra en la Comuniadad Autónoma Vasca tiene también repercusiones metodológicas en el presente trabajo.

Veamos el porque; la estadística elaborada por la Administración central ignora frecuentemente la realidad de las distintas nacionalidades y regiones del Estado, por un lado, y actualmente el Gobierno Vasco y la 
Diputación Foral de Navarza trabajan, cada una por sepatado, los datos referentes a los territorios de su competencia, por otro lado. A resultas de todo ello, la obtención de datos homogéneos para el total de Euskal Hertia Sur se hace muy difícil, cuando no imposible del todo.

\section{EVOLUCION DE LA POBLACION ECONOMICAMENTE ACTIVA}

Indicaremos en una primera precisión que la población económicamente activa comprende a los ocupados (en sentido estricto), a los activos marginales y a los parados.

Pasando a los datos, tenemos que en la serie de 3 años observada para el conjunto de Euskal Herria Sur en la tabla 1, es posible adivinar un estancamiento en las cifras de población activa, a la vez que destaca la fuette variabilidad interanual: $983,5,988,1$ y 983,2 miles de activos.

Para las tres provincias que componen la Comunidad Autónoma de Euskadi, la serie de datos es ya mayor. A nivel global podernos observar un crecimiento mantenido que va desde 801,3 hasta 812,8 miles en la serie de años estudiada, si bien en 1981 se da un fuerte crecimiento que en el siguiente año vuelve a ser neutralizado, para volver a tecuperarse parcialmente al menos en $1983 .{ }^{1}$

$\mathrm{Si}$ descendemos al nivel provincial en nuestros análisis, tenemos que tanto en Alava, como en Guipúzcoa y en Vizcaya, se da un incremento de la población activa en el total de la serie. Ahora bien, el comportamiento varía de una a otra provincia en los distintos años: Álava da su cifra más alta en $1981(102,0)$, Guipúzcoa la alcanza en $1982(270,1)$ y Vizcaya Io hace en 1983 (primer semestre: 448,4).

Comparativamente, a nivel de Estado podemos observar la misma tendencia ascendente entre los años 1979 y 1982, si bien en 1980 y 1981 las cifras etan inferiotes a las de 1979. En términos relativos, obsetvando el período que va desde 1979 hasta 1983, el mayor crecimiento de la población activa se da en Álava con un 103,1, partiendo de 100 en la primeta fecha; en Vizcaya es algo inferior $(101,4)$, mientras que en Guipúzcoa se mantiene la población activa prácticamente estacionaria $(100,7)$. En el cómputo para la Comunidad Autónoma se repite la misma cifra que para Vizcaya $(101,4)$. Para el conjunto del Estado vemos que la población activa

1. Gobiemo Vasco. Dirección de Estatística, Censo de Población y Vivienda 1981 de la Comunidad Autónoma de Euskadi. Estructura de la Población, 1983. 
«Papers»: Revista de Sociologia

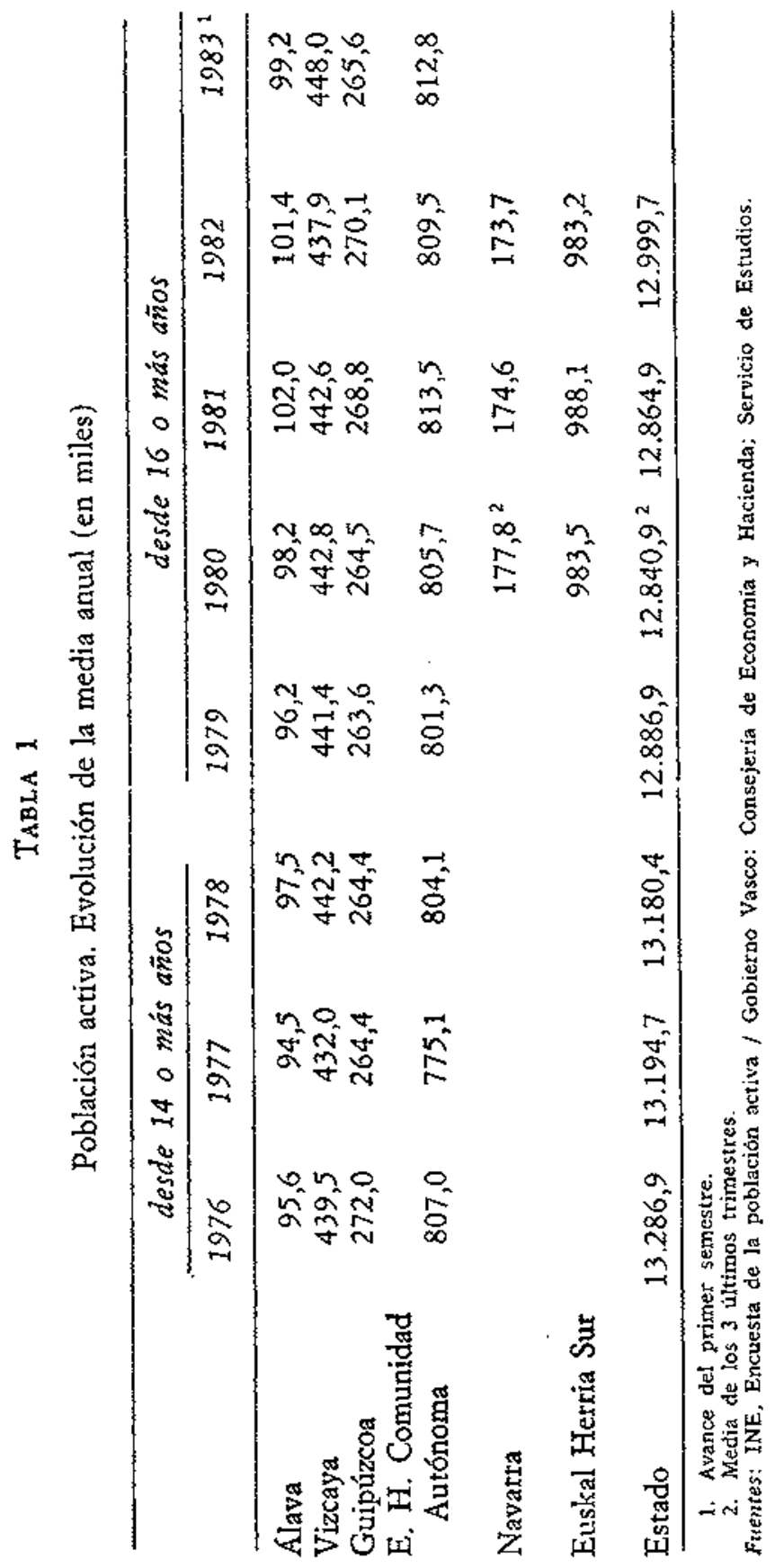


Mercado de trabajo y fuetzas sociales en Euskal Herria Sur TABLA 1 bis (indices)

\begin{tabular}{lrrrr} 
& 1979 & 1980 & 1982 & $1983^{2}$ \\
\hline Alava & 100 & & 105,4 & 103,1 \\
Vizcaya & 100 & & 99,2 & 101,4 \\
Guipúzcoa & 100 & & 102,4 & 100,7 \\
$\begin{array}{l}\text { E. H. Comunidad } \\
\quad \text { Autónoma }\end{array}$ & 100 & & 101,0 & 101,4 \\
Navarra & & 100 & 97,6 & \\
Euskal Herria Sur & & 100 & 99,9 \\
Estado & 100 & & 97,8 \\
\hline
\end{tabular}

1. Avance del primer semestre.

se queda en 97,8 para 1982 , habiendo partido también de 100 en 1979.

Esta diferencia entre la evolución de las poblaciones activas de Euskadi y del total del Estado, para un período de 3-4 años, vamos a analizarla ahora con mayor perspectiva temporal, dejando a un lado las características provinciales.

Como podemos ver en la tabla 2, tanto en cifras absolutas como en crecimiento relativo, el crecimiento de la población activa (PA) vasca es muy importante en comparación con la del Estado hasta 1980. En el perío. do 1980-82 podemos observar un estancamiento para el conjunto de Euskal Herria Sur, frente al mencionado crecimiento para las Vascongadas entre 1979-83, citado anteriormente, y frente también al sostenido incremento observado en el conjunto del Estado.

Resumiendo todo lo dicho hasta el momento tenemos que la PA ha experimentado un crecimiento mucho más importante en Euskal Herria Sur que en el total del Estado desde 1950 hasta 1980, pero, a partir de esta fecha, en Euskadi Sur se da un estancamiento o fuerte ralentización en tal crecimiento, mientras que a nivel de Estado se mantiene persistentemente una moderada alza.

Es evidente que esta repentina intertupción del crecimiento de la PA vasca ha de ser puesto en relación con la tan repetida crisis económica, a través de la evolución de la población. 
«Dapers»: Revista de Sociologia

TABLA 2

Evolución de la población activa (en miles e índices)

\begin{tabular}{lrrrrr} 
& 1950 & 1960 & 1970 & 1980 & 1982 \\
\hline Euskal Hetria Sur & 592,8 & 680,3 & 824,8 & 983,5 & 983,2 \\
Estado & $10.793,1$ & $11.816,6$ & $12.732,2$ & $12.840,9$ & $12.999,7$ \\
Euskal Herria Sur & 100 & 114,7 & 139,1 & 165,9 & 165,9 \\
Estado & 100 & 109,4 & 117,9 & 118,9 & 120,4 \\
\hline
\end{tabular}

Fuente: El desempleo en el Pals Vasco, Caja Laboral Popular / Enctesta de Población Activa. INE / Servicio de Estudios de la Consejería de Economía y Hacienda. Gobierno Vasco.

\section{Algunos datos Relacionados con la población activa}

La profunda crisis económica iniciada en 1973.74 en nuestro país a podido ver retrasados sus efectos sobre la demografía, pero su ininterrumpida agudización ha conseguido imponer unas nuevas pautas de compottamiento demográfico.

«Las circunstancias demográficas actuales de la Comunidad Autónoma del País Vasco parecen caracterizarse por una crisis a todos los niveles. Las tasas brutas de natalidad -obtenidas a partir de datos provisionalesse encuentran en niveles bajísimos oscilando del 12 al 13,5\%, según el Territorio (o provincia): dichas tasas eran incluso infetiores a las del Estado (estimada en 1981 en un $13,8 \%$ ).

"La mortalidad muestra una ligera tendencia al aumento, llegando casi al $8 \%$ en 1981. Con ello se produce una reducción drástica del crecimiento vegetativo, que se situaría en un $0,4 \%$ anual...

»La migración neta muestra, igualmente, una tendencia recesiva, con un número de salidas por encima del de entradas en los últimos años. De esta forma, todos los componentes del crecimiento demográfico se aúnan para contribuir al estancamiento de la población, incluso con un ligero descenso a corto plazo. Y es difícil imaginar una brusca inversión en las tendencias en los próximos años.» $)^{2}$

2. Gobierno Vasco. Dirección de Estadistica, Evolución de la población. Período 1900-1981, junio 1982. 
TABLA 3

Evolución de la población de hecho y de las tasas de crecimiento intercensal (TCI) en la Comunidad Autónoma del País Vasco y en el Estado español

\begin{tabular}{rrrrrr}
\hline & \multicolumn{2}{c}{ CAPV } & & \multicolumn{2}{c}{ Estado español } \\
\cline { 2 - 3 } \cline { 5 - 6 } Año & Población & TCI \% & & Población & TCI \% \\
\hline 1787 & 308.157 & & 10.268 .150 & \\
1860 & 429.139 & 0,45 & 15.645 .072 & 0,58 \\
1877 & 450.699 & 0,29 & 16.622 .100 & 0,36 \\
1887 & 510.408 & 1,25 & 17.549 .608 & 0,54 \\
1900 & 603.596 & 1,30 & 18.616 .630 & 0,46 \\
1910 & 673.788 & 1,11 & 19.990 .909 & 0,71 \\
1920 & 766.775 & 1,30 & 21.388 .551 & 0,68 \\
1930 & 891.710 & 1,52 & 23.677 .095 & 1,02 \\
1940 & 955.764 & 0,70 & 26.014 .278 & 0,95 \\
1950 & 1.061 .240 & 1,05 & 28.117 .873 & 0,78 \\
1960 & 1.371 .654 & 2,60 & 30.582 .936 & 0,84 \\
1970 & 1.878 .636 & 3,20 & 33.956 .376 & 1,05 \\
1975 & 2.072 .430 & 1,98 & 36.026 .319 & 1,19 \\
1981 & 2.134 .967 & 0,58 & 37.674 .594 & 0,87 \\
\hline
\end{tabular}

GRÁfYCo 1

Evolución de la población de hecho en la CAPV

Población (millones)

${ }_{1,0}^{2,0}=$

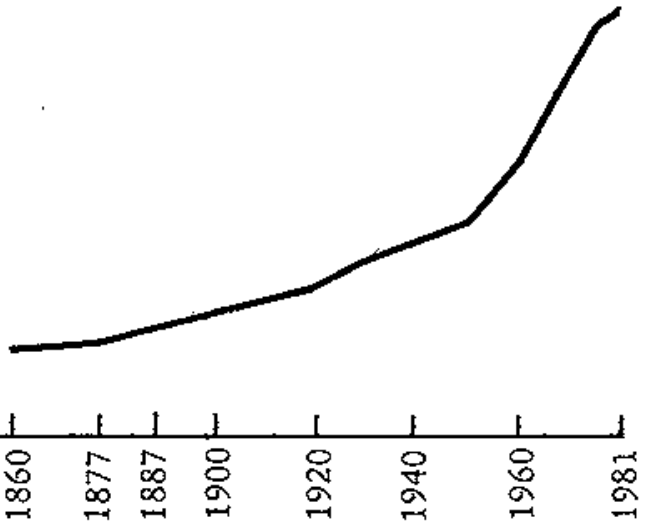

Fuente: Gabierno Vasco. Direccion de Estadística. Evolución de la población. Periodo 19001981. 
«Papets»: Revista de Sociologia

Como podemos ver en la tabla 3, las tasas de crecimiento intercensal de la población, alcanzadas entre 1975 y 1981, son las más bajas registradas desde 1877, es decir, las más bajas en los últimos 100 ar̃os. Si aquí incluyésemos las cifras correspondientes a Navarra, posiblemente la caída de las tasas no fuese tan rotunda, pero se mantendría su importancia.

Ahora bien, pongamos este hecho en relación con la ralentización del crecimiento de la PA. Es evidente que una reducción en la tasa de natalidad, como la anteriormente citada para la Comunidad Autónoma Vasca, no es posible que tenga una infuencia directa en la PA antes de que transcurran 16 años, en el caso de mantenerse la legislación actual y de no mediar otros fenómenos perturbadores. Sin embargo, esto ha de ser tenido también en cuenta para una previsión de futuro, aunque no baya de ser posiblemente el factor decisivo.

La mortalidad tampoco puede explicarnos el estancamiento de la PA a partir de 1980; por tanto, nos queda solamente el fenómeno migratorio.

Segun podemos observar en la tabla 4, a partir de 1978 los saldos migratorios de Euskal Herria Sur manifiestan un punto de inflexión, preponderando la emigración sobre Ia inmigración, hecho desconocido para el

\section{TABLA 4}

Evolución de los saldos migratorios

\begin{tabular}{rrrrrr}
\hline Año & Alava & Guipuzzoa & Navarra & Vizcaya & $\begin{array}{c}\text { Euskal } \\
\text { Herria } \\
\text { Sur }\end{array}$ \\
\hline 1965 & 4.000 & 6.870 & - & 18.892 & - \\
1970 & 2.585 & 1.794 & 1.994 & 6.460 & 12.833 \\
1975 & 2.085 & 756 & 600 & 7.194 & 10.635 \\
1976 & 676 & -1.480 & 563 & 949 & 708 \\
1977 & 1.182 & -1.663 & 1.524 & -29 & 1.014 \\
1978 & 979 & -3.506 & 537 & -4.219 & -6.209 \\
1979 & 858 & -5.044 & 316 & -6.060 & -9.930 \\
1980 & 513 & -4.663 & 400 & -6.311 & -10.061 \\
1981 & 570 & -1.945 & 246 & -3.677 & -4.806 \\
1982 & 580 & -2.735 & 682 & -4.203 & -5.676 \\
\hline
\end{tabular}

Fuente: Caja Laboral Popular. El desempleo en el Pais Vasco y Economía vasca. Intorme 1982. 
conjunto del país desde la década de 1901-1910 (a nivel provincial esto sí se da en determinados períodos). ${ }^{3}$

Evidentemente, la búsqueda de un puesto de trabajo fue lo que atrajo oleadas de inmigrantes, y en la carencia del mismo se encuentra la razón de la presente emigración.

Es destacable que la reciente emigración aparenta haber tocado techo en los años 1979 y 1980 con unos saldos de 9.930 y 10.061 salidas de pobladores respectivamente, para después mantenerse constante en 1981 y 1982 con valores inferiores ( 4.806 y 5.676 , respectivamente). Es preciso señalar que los totales referidos a Euskal Herria Sur no son teales porque tienen incluidos la emigración interprovincial entre Álava, Guipúzcoa, Navarra y Vizcaya, pero pueden darnos una idea de la trayectoria de los movimientos migratorios existentes.

Esta salida de población hacia otras tierras afecta muy directamente a las cifras de $P A$, aunque la emigración se pueda ver compensada por el crecimiento vegetativo y la PA se mantenga o incluso crezca ligeramente.

No resulta difícil imaginar las múltiples perturbaciones en la estructura socio-económica que todo esto puede ocasionar: cambio en las expectativas de consumo y de vida de la población, constreñimiento de la demanda de bienes de consumo, inseguridad en el puesto de trabajo, escasas expectativas profesionales en los jóvenes, aplazamiento de la normalización y consolidación de la vida de pareja, crisis de los valores de un sistema basado en la expansión... Todas estas y otras muchas alteraciones acontecidas en el tejido social obviamente han de ocasionat, a su vez, una obsoletización de Ios movimientos sociales y cauces tradicionales que intentaban combatir las deficiencias - coyunturales y estructurales- del sistema vigente.

Quede señalada la transcendencia de estos hechos y la conveniencia de impulsar estudios que permitan aprehender debidamente esta importante época de transición que nos tiene por testigos y protagonistas.

\section{LA POBLACIÓN ACTIVA FEMENINA}

Una vez vista la evolución de la población activa en su conjunto, tomemos ahora en consideración la desagtegación de la misma por sexos.

Según podemos contemplar en la tabla 5 , el $70,3 \%$ de la PA, en 1982, eran varones y el $29,6 \%$ restante eran mujeres. Esto para el total de

3. Llera Ramo, Francisco José, La estructura social del Pais Vosco, «Revista In. ternacional de Sociologías. Separata, Madrid, octubre-diciembre 1982. 
«Papers»: Revista de Sociologia

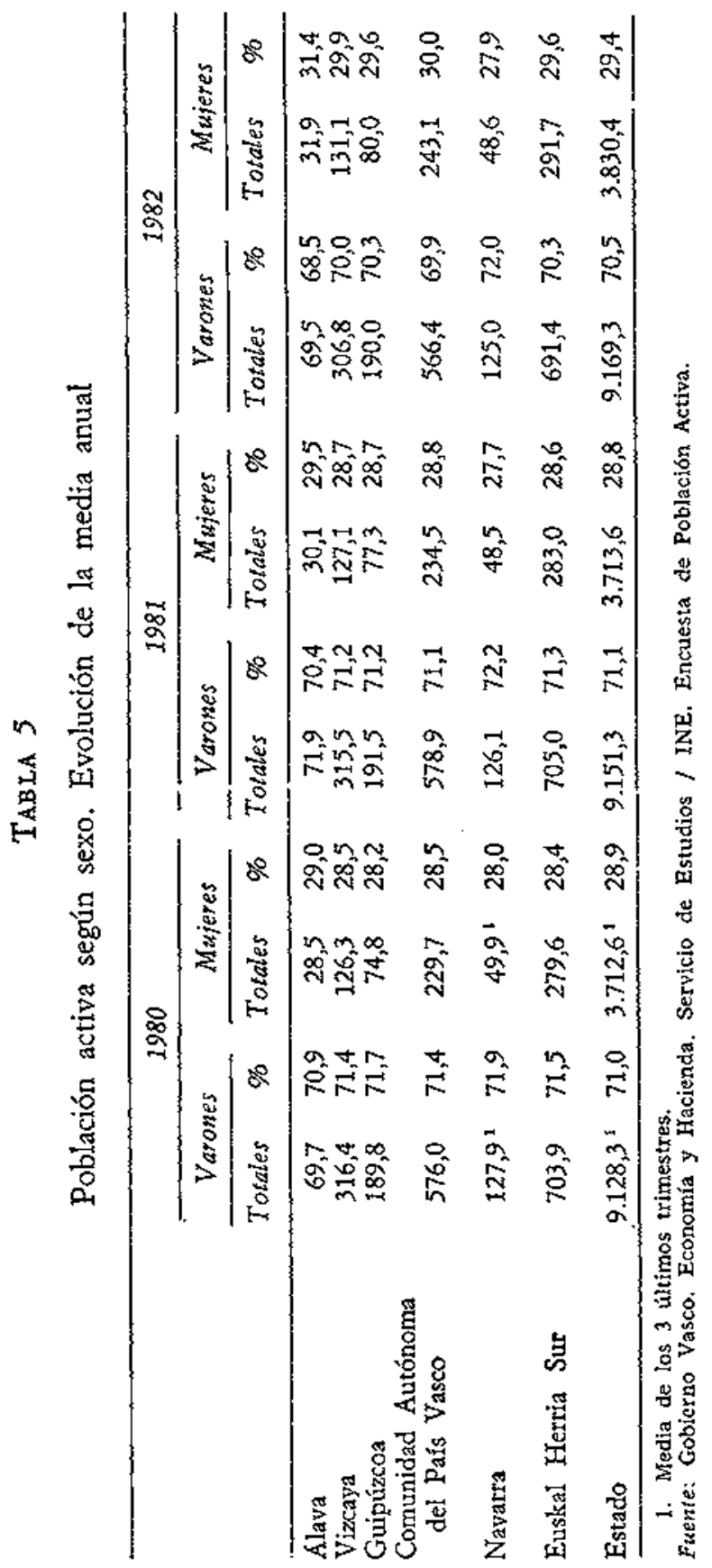


Mexcado de trabajo y fuerzas sociales en Euskal Herria Sur

TABLA 6

Evolución de la tasa de actividad femenina * (en \%)

\begin{tabular}{|c|c|c|c|c|c|c|c|c|c|}
\hline Años & $\begin{array}{c}\text { Alemani } \\
\text { R.F. }\end{array}$ & Bêlgica & Francia & $\begin{array}{l}\text { Dina- } \\
\text { marca }\end{array}$ & Italia & Irlanda & $\begin{array}{l}\text { Reino } \\
\text { Unido }\end{array}$ & $\begin{array}{l}\text { Pais } \\
\text { Vasco }\end{array}$ & España \\
\hline 1950 & 38,9 & 30,3 & - & 40,8 & 一 & 31,8 & 36,1 & - & - \\
\hline .1955 & 40,9 & 30,0 & 一 & 41,3 & - & - & 38,5 & - & - \\
\hline 1960 & 41,8 & 30,1 & 一 & 35,3 & 32,7 & 28,2 & 38,3 & - & - \\
\hline 1965 & 40,4 & 30,9 & - & 40,4 & 26,7 & 29,1 & 39,8 & 一 & $\leftarrow$ \\
\hline 1970 & 38,7 & 32,3 & 41,0 & 47,7 & 24,9 & - & 40,5 & 一 & 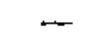 \\
\hline 1971 & 38,5 & 32,7 & 38,1 & 48,6 & 24,7 & 28,0 & 40,2 & 一 & 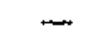 \\
\hline 1972 & 38,3 & 33,0 & 38,4 & 49,8 & 23,9 & $\ldots$ & 40,7 & - & 25,9 \\
\hline 1973 & 38,5 & 33,9 & 39,5 & 50,4 & 24,5 & + & 42,2 & - & 28,3 \\
\hline 1974 & 38,4 & 34,6 & 39,8 & 51,1 & 24,9 & - & 43,0 & - & 28,8 \\
\hline 1975 & 37,8 & 35,0 & 39,8 & 51,3 & 25,2 & - & 43,4 & - & 28,1 \\
\hline 1976 & - & - & $\rightarrow$ & - & - & - & - & 27,8 & 27,4 \\
\hline 1977 & r & - & - & - & - & - & - & 27,5 & 27,5 \\
\hline 1978 & - & - & - & - & $m$ & - & 一 & 27,4 & 26,9 \\
\hline 1979 & - & - & $\ldots$ & - & - & - & 一 & 28,2 & 26,7 \\
\hline
\end{tabular}

* Calculada sobre la población femenina mayor de 14 años.

Fuente: Eurostat y EPA / Tomado de la Caja Laboral Popular, El desempleo en el País Vasco.

Euskal Herria Sur. Pata el total del Estado tenemos unas cifras muy similares: 70,5 y $29,4 \%$, respectivamente.

Si tomamos en cuenta únicamente la Comunidad Autónoma de Euskadi, la cifra porcentual de mujeres incluidas en la PA es ligeramente superior: $30 \%$.

Como se puede apteciar en la tabla 6 , la incorporación de la mujer al metcado de trabajo permanece bastante alejada de lo habitual en la mayoría de los países europeos, sobre todo de los tnás adelantados. Así, tenemos que la tasa de actividad femenina es del 27,8 \% para el País Vasco Sur y del $27,4 \%$ para España en 1976, mientras que únicamente Italia está por debajo de esa cifra en 1975. El resto de estados considerados está por encima y en el caso del Reino Unidos $(43,4 \%)$ Ia diferencia es notoria.

Esto puede ser muy significativo de la mentalidad que sobre la función social de la mujer tiene la sociedad y la propia mujer.

Aceptamos como indiscutible que una mayor incorporación de la mujer al proceso productivo puede ser considerado como uno de los indicadores del grado de modernización de la sociedad. Ahora bien, ese mismo indicador no debe ser necesariamente interpretado como teflejo de una mayor liberación femenina. Veamos con más extensión este hecho. 
La incorporación de la mujer al mercado de trabajo implica, o por lo menos propicia, un cambio en las funciones familiates, tendiendo a que sean repartidas las faenas propias del hogar. La profundidad o no del cambio en los toles hogareños depende de factores psicológicos individuales y características familiares que no vienen a cuento. Ahora bien, aparte de lo mencionado se puede avanzar la hipótesis de "persistencia de la subor. dinación femenina en el puesto de trabajo». Es decir, la mujer, según esto, encuentra respuestas a sus demandas de trabajo en un nivel profesional inferior a to habitual en el hombre. Frecuentes son los casos en que se niega el acceso a ciertos puestos por el hecho de ser mujer, así como otros en que se prefiere a las mujeres por que es posible retribuirles con salarios inferiotes.

Llegamos ahora a plantearnos estos hechos en conexión con el movimiento feminista y preguntatnos: ¿qué incidencia puede tener la incorporación más o menos intensa de la mujer al mundo del trabajo en la propia condición de la mujer y en sus reivindicaciones como colectivo social oprimido? ¿La incorporación al puesto de trabajo provocatá en la mujer el sentimiento de marginación y opresión que permaneciendo al frente de las tareas hogareñas ha sido difícil que sintiera? He aquí dos cuestiones que esperan respuesta.

Pero atendamos ahora a la evolución de la incorporación de la mujer a la PA y tenemos que, entre 1976 y 1979, en Euskal Herria Sur se da un ligeto incremento (de 27,8 a $28,2 \%$ ) en la tasa de actividad femenina, frente al descenso que para ese mismo período se observa a nivel de Estado (de 27,4 a $26,7 \%$ ) (tabla 6). Avanzando en el tiempo, vemos en la tabla 5 que también prosigue el aumento expresado en Euskal Herria Sur (aunque los datos entre ambos cuadros no son homogéneos). A nivel de Estado también se da un aumento, aunque menor.

Si recordamos que a partir de 1980 la PA se estancaba o crecía muy lentamente (tabla 2) y advertimos ahora que el peso femenino va aumentando, se deduce que el parón en el crecimiento de la PA se debe funda. mentalmente a la retirada de los hombres del mercado de trabajo.

«La caída en la tasa de actividad de los varones ha venido influen. ciada, aparte de por el factor migratorio, por el aumento de las tasas de escolarización. La desaceleración en la caída de la tasa global ha sido con. trarrestada en parte por el factor de signo contrario manifestado por la cada vez mayor incorporación de la mujer al trabajo.

»La oferta de trabajo manifiesta, por tanto, fuertes oscilaciones respecto a la situación de la demanda. Ante unas posibilidades escasas de en. contrar un puesto de trabajo por su escasez y progresiva reducción en el 
metcado, la oferta se retrae, no significando esto que la población activa sea inferior, sino que no se manifiesta como tal ante las escasas perspectivas de encontrar una colocación. $\rangle^{4}$

De acuerdo con esta opinión, en una época de crisis como la actual es preciso tener en cuenta tal elasticidad de la oferta de mano de obra y la repercusión que sobre las cifras de PA puede suponer. Se echan en falta estudios sobre el peso relativo que tienen factores tales como las recientes corrientes migratorias y el posible retraimiento de la oferta de tmano de obra en el estancamiento de la evolución de la PA observado a partir de 1980 .

\section{IMPORTANCIA CUALITATIVA DE LA EMIGRACION}

Se ha hecho ya referencia al peso cuantitativo que la reciente emigración vasca supone en la evolución anual de la PA. Aquí podemos observar nuevamente la progresión ascendente que tal fenómeno ha mantenido entre 1976 y 1980 , pasando de 18.419 a 33.826 emigrados en esos años respectivamente, en lo que atañe a la Comuridad Autónoma de Euskadi. Es decir, la emigración casi se ha duplicado en el petíodo considerado a nivel de Comunidad Autónoma y otro tanto acontece a nivel de cada teritotio histótico o provincia. Abordamos seguidamente una aproximación cualitativa de la emigración en el mismo período.

Vemos en la tabla 7 una distribución de la emigración según el territorio de salida, el hecho de no estar incluido en la PA, el estar incluido en la misma - con la especificación del sector de actividad al que se pertenece- $y$, por último, el ser analfabeto (aunque esta categoría es irrelevante dentro del conjunto porque en ningún año de los estudiados llega a alcanzar el $1 \%$ ). Según este cuadro, las personas emigradas pertenecen más a la categoría de los no activos (casi los 2 tercios) que a la de activos (algo más de 1 tercio).

Consideremos la familia típica a aquella que se compone de matrimonio y dos hijos, y supongamos que únicamente uno de los cónyuges pertenezca a la categoría económica de activo - ya que no es muy probable que si ambos cónyuges son activos, se queden los dos sin empleo, hecho que suponemos sea la principal causa de emigración en estos tiempos de crisis, principalmente.

4. Caja Laboral Populat, El desempleo en el País Vasco, 1982, p. 47. 


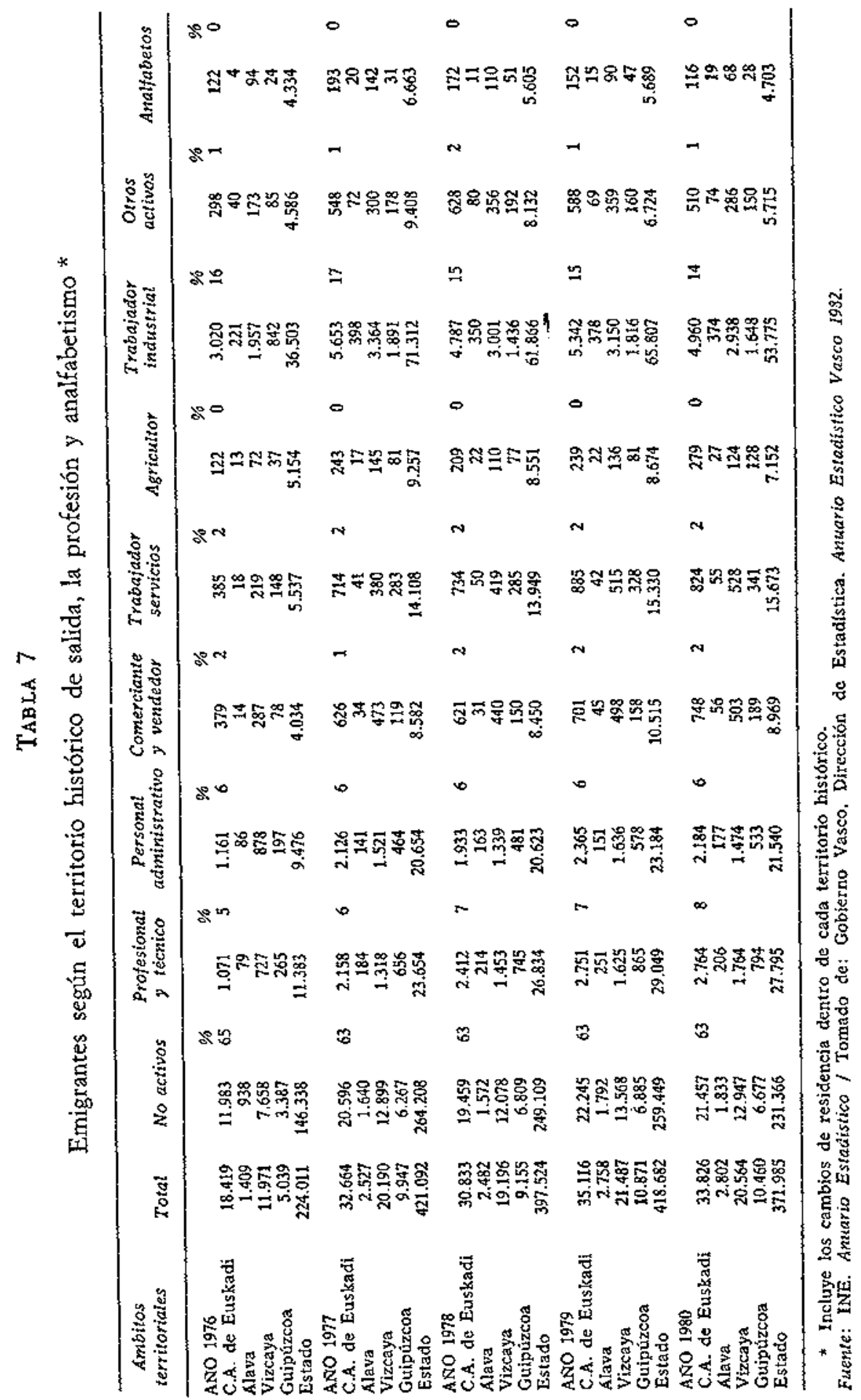


Mercado de trabajo y fuetzas sociales en Euskal Herria Sur

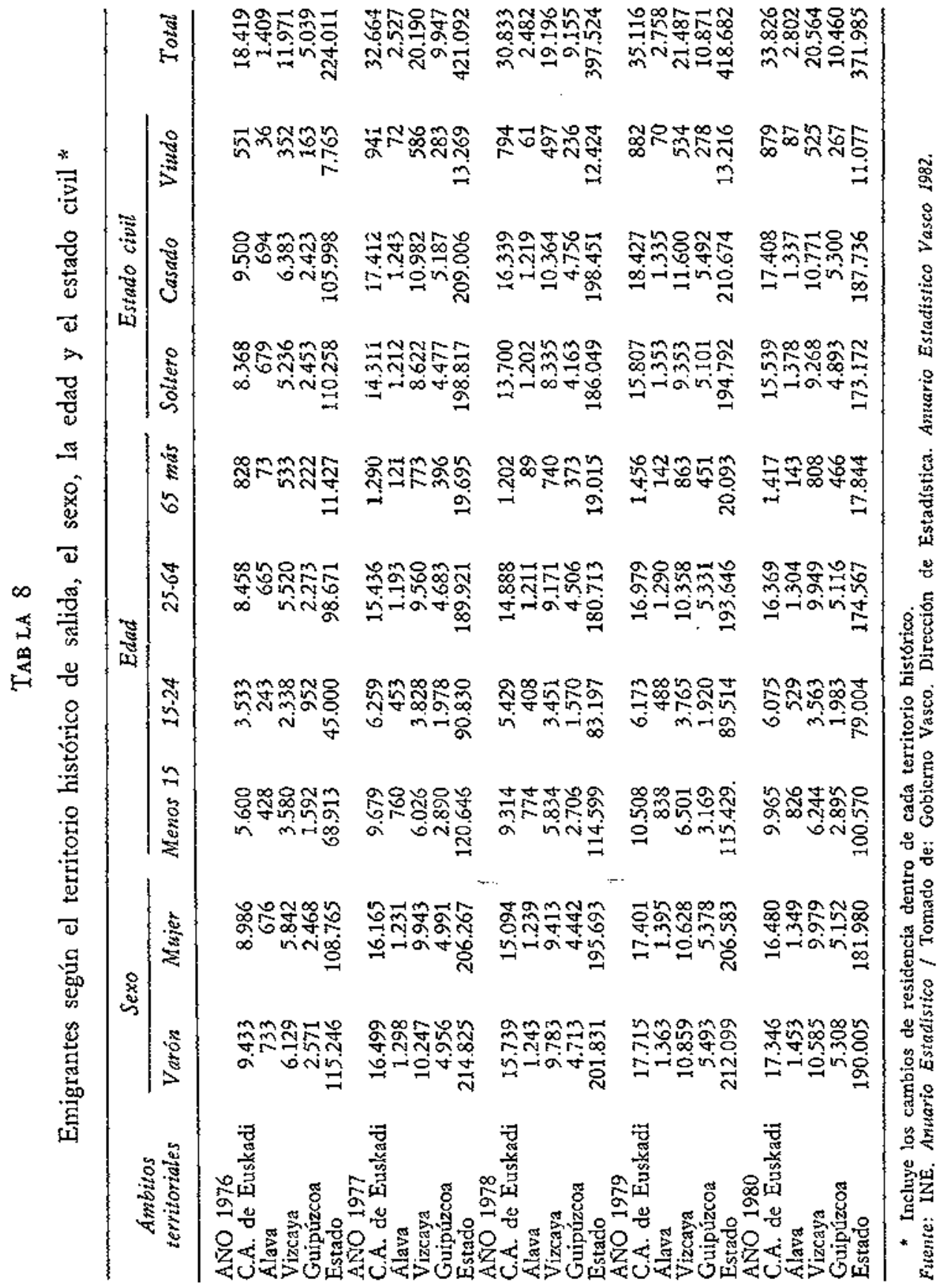


«Papers»: Revista de Sociologia

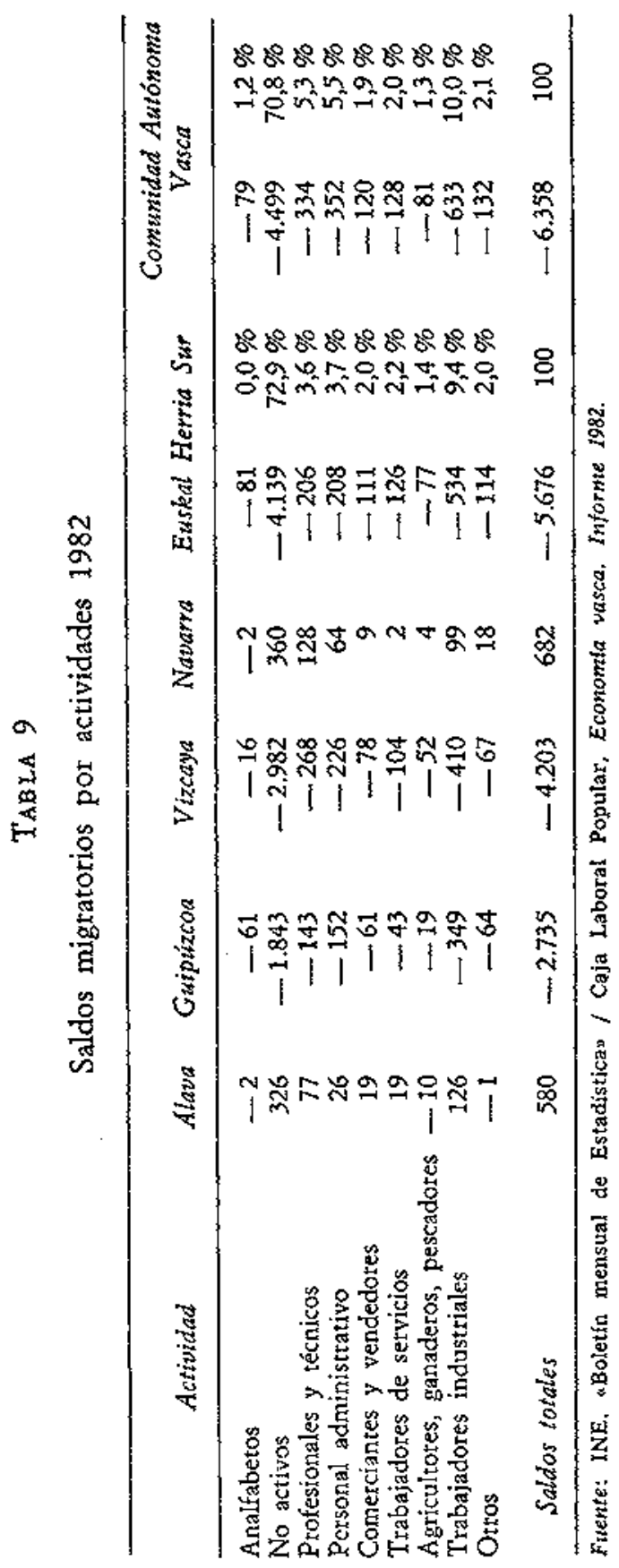


Partiendo de estas hipótesis interpretativas, concluiremos que los activos emaigrados (más de un tercio) superan con creces a los activos de la hipótesis emigratoria (un cuarto).

Una vez visto esto recurrimos al estudio del sexo, edad y estado civil de los emigrados (tabla 8). Según el sexo vemos que varones y mujeres emigrados se hallan muy equilibrados, si bien estas últimas son en todos los años menos que los hombres: 49,1 frente a $50,9 \%$, respectivamente, para la media de todos los años considerados. Estas cifras están en conso nancia con las resultantes del censo de población de la Comunidad Autónoma Vasca a 1 de marzo de 1981:

«(..) el ratio entre sexos que empieza siendo de 1,06 varones por mujer a los 0.4 años, pasa a 1,02 a los 15.19 , manteniéndose aproximadamente constante hasta los 55 años; a partir de entonces, el ratio desciende rápidamente: 0,97 a los $55-59$ años, 0,67 a $70-74(\ldots){ }^{5}{ }^{5}$

En nuestro caso, los emigrantes son preferentemente jóvenes (sólo suponen el $5 \%$ a partir de los 65 años), por tanto, podemos concluir que los emigrantes se ajustan bastante bien a la estructura por sexos de la población, y tomando esta variable no parece que se deduzca ninguna consecuencia que ayude en la explicación del desajuste de la PA emigrada con la que hipotéticamente suponíamos podía emigrar.

Si consideramos ahora la tabla 9 vemos que, para 1982, los saldos migratorios -es decir, suma algebraica de inmigrantes y emigrados- tanto para el conjunto de Euskal Herria Sur como para la Comunidad Autónoma Vasca solamente los porcentajes de emigración de no-activos se acercan a la hipótesis emigratoria propuesta. No podemos detenernos más en este aspecto cuyo interés para investigaciones más especializadas es bien manifiesto.

Retornando a la tabla $\%$, podemos observar que los porcentajes más elevados de emigrados, de entre los activos, se da para los trabajadores undustriales; no obstante, parece darse un descenso en la importancia relativa de la emigración de este grupo, pasando del $16 \%$ en 1976 al $14 \%$ en 1980. Lo contrario acontece en el segundo grupo de activos por su importancia relativa: los profesionales y técnicos emigrados suponen el $5 \%$ en 1976 y ascienden hasta el $8 \%$ en 1980. ¿Quiere esto decir que la Comu nidad Autónoma Vasca padece una creciente sangría en el sector de población activa más cualificado?

5. Gobierno Vasco. Dirección de Estadística, Censo de población y Vivienda..., p. 298. 
"Papers»: Revista de Sociologia

Yendo a los datos de 1982 (tabla 9) no podemos establecer comparaciones directas con los presentados en la tabla 7 debido a que corresponden a saldos migratorios en la primera y a cifras de emigrados en esta última. Sin embargo, no somos los únicos en manifestar tal temor, ya que también el informe sobre 1982 de Caja Laboral Popular hace un comentario en este sentido:

«(...) La clasificación por actividades de los saldos migratorios arroja algunas informaciones de interés. Entre éstas destaca la salida del grupo de profesionales y técnicos, no muy importante en términos cuantitativos pero sí en cuanto a su significación como exponente de un fenómeno de descapitalización humana.» ${ }^{6}$

Queda apuntada la importancia de un estudio más profundo de este hecho, tanto por lo que ello puede aportar a un mejor conocimiento de la realidad y sus entresijos, como para poder actuar en evitación de unos flujos emigratorios gravosos y lamentables tanto humana como económicamente.

\section{DISTRIBUCION SECTORIAL DE LA POBLACION ACTIVA}

Pasamos seguidamente a describir la distribución sectorial de la población activa ocupada en el periodo comprendido entre 1960 y 1982 (tabla 10). Tanto para Euskal Herria Sur como para el Estado en su conjunto, se ha dado un fuerte desplazamiento intersectorial de la PA. Así, tenemos que en Euskal Herria Sur el porcentaje de PA ocupada en la agricultura era del $21,8 \%$ en 1960 , mientras que en 1982 sólo suponía un $8,1 \%$. Es decir, se perdieton 13,7 puntos. De modo más agudizado aún aparece este fenómeno para todo el Estado en el mismo período de tiempo con una pérdida de 23,7 puntos (de 41,7 a $18,0 \%$ ).

6. Caja Laboral Populat, Economia Vasca. Intome 1982, p. 524. 
Mercado de trabajo y fuerzas sociales en Euskal Herria Sur

TaBla 10

Distribución sectorial de la población activa ocupada (en \%)

\begin{tabular}{cccccccc}
\hline & \multicolumn{3}{c}{ País Vasco Sur } & & \multicolumn{3}{c}{ España } \\
\cline { 2 - 4 } \cline { 5 - 7 } Año & Agricultura & Industria & Servicios & Agricultura & Industria & Servicios \\
\hline 1960 & 21,8 & 50,7 & 27,5 & & 41,7 & 31,8 & 26,5 \\
$1970 *$ & 12,1 & 52,2 & 34,6 & 29,1 & 37,3 & 33,6 \\
1975 & 10,3 & 51,9 & 37,8 & & 21,7 & 37,7 & 40,6 \\
1979 & 8,0 & 48,9 & 43,1 & 19,6 & 36,3 & 44,2 \\
1980 & 8,8 & 50,0 & 41,1 & 18,9 & 36,0 & 45,2 \\
1982 & 8,1 & 47,0 & 44,8 & 18,0 & 34,3 & 47,7 \\
\hline
\end{tabular}

* Para el Pass Vaseo datos de 1971.

Fuentes: Caja Laboral Popular, El desempleo en el Pais Vasco / Cámaras de Comercio vascas, Boletin de Coyuntura y Estadístice del Pais Vasco.

Observando ahora la industria vemos que, tanto en el País Vasco Sur como en el Estado, se da un incremento potcentual en 1970 y 1975 con respecto a 1960 pata luego volver a descender en ambos casos. El incremento señalado es coincidente en el tiempo con los años de fuerte expansión económica, del mismo modo que el posterior descenso vuelve a coincidir con el inicio de la crisis actual. A destacar que en Euskal Herria Sur el descenso porcentual de la mano de obra dedicada a la industria es mayor que en el Estado: en el caso vasco cae por debajo de la cifra alcanzada en $1960(1982=47,0 \%$ y $1960=50,7 \%)$. En el Estado no llega a caer por debajo de la cifta de $1960=31,8 \%(1982=34,3 \%)$.

En el sector servicios se da un crecimiento para ambos casos en el mismo período, siendo mayor en el conjunto estatal $(21,2$ puntos frente a 17,3 para Euskal Herria Sur).

De todo ello podemos deducir que la distribución sectorial de la población activa está más asentada en el País Vasco Sur que en el Estado en el período considerado (1960-1982). Ello es muy razonable ya que el proceso de industrialización en el Pais Vasco Sut - y sobre todo en Vizcaya y Guipúzcoa- estaba consolidado para esas fechas, mientras que amplias zonas del Estado se han industrializado precisamente en esas fechas.

Contemplemos abora la distribución sectorial de la población ocupada a la luz de las citras de los principales paises europeos (tabla 11). Seguimos para ello un informe de Caja Laboral, en el que se indica que la distribución de la PA ocupada «por los tres sectores básicos revela una participación mayoritaria, y en general superior al $50 \%$ del total, del sector de los servicios en el contexto europeo tratado, a excepción de Italia, el 
Estado español, y de forma acusada en el País Vasco. La dotación de empleos en el sector (servicios), con tespecto a la industria, guatda un fuerte desequilibrio en nuestro país si lo comparamos con la situación europea, con un peso excesivo en el sector industrial.

"La tendencia durante los ocho años considerados tevela ya un fuerte desplazamiento hacia el sector terciario, factor lógico si se tiene en cuenta el inctemento durante ese período en la demanda de servicios debidos al aumento de los niveles de renta y la creciente terciarización de las economías en cutestión; sin embargo, destaca el todavía débil peso que este sector ha logrado en la economía vasca. Paralelamente, la industria cede terteno de forma importante en la participación de sus empleos, al ir desplazando parte de sus activos hacia aquel sector, debido a sus mayores incrementos de productividad que le conferen mayor capacidad de generación de empleos que en las épocas precedentes al inicio de la crisis económica. En el País Vasco esta disminución apenas se materializa en dos puntos porcentuales.

\section{TABLA 11}

Distribución sectorial de la población activa ocupada (en \%)

\begin{tabular}{|c|c|c|c|c|c|c|c|c|c|}
\hline \multirow[b]{2}{*}{ Paises } & \multicolumn{3}{|c|}{ Agricultura } & \multicolumn{3}{|c|}{ Industria } & \multicolumn{3}{|c|}{ Servicios } \\
\hline & 70 & 75 & 78 & 70 & 75 & 78 & 70 & 75 & 78 \\
\hline Austria & 18,8 & 12,5 & 10,9 & 40,3 & 40,9 & 40,6 & 40,8 & 46,6 & 48,5 \\
\hline Bélgica & 4,7 & 3,6 & 3,2 & 43,2 & 39,8 & 36,6 & 52,0 & 56,5 & 60,2 \\
\hline Holanda & 7,2 & 6,6 & 6,2 & 38,6 & 34,6 & 32,5 & 54,2 & 58,9 & 61,3 \\
\hline Suiza & 8,6 & 7,9 & 7,6 & 46,0 & 41,5 & 39,9 & 45,4 & 50,6 & 52,5 \\
\hline Alemania R.F. & 8,6 & 7,4 & 6,5 & 49,3 & 46,0 & 45,0 & 42,1 & 46,6 & 48,5 \\
\hline Francia & 13,9 & 10,2 & 9,2 & 39,7 & 38,8 & 37,1 & 46,4 & 51,0 & 53,8 \\
\hline Italia & 20,3 & 16,8 & 15,5 & 39,7 & 39,3 & 38,3 & 40,1 & 44,0 & 46,2 \\
\hline Reino Unido & 3,2 & 2,7 & 2,7 & 44,8 & 40,7 & 39,6 & 52,0 & 56,6 & 57,8 \\
\hline & 29,5 & 22,1 & 20,2 & 37 & 38 & 37,3 & 33,2 & 39,7 & 42,5 \\
\hline País Vasco & 12,1 & 10,3 & 9,0 & 52,2 & 51,9 & 50,2 & 34,6 & 37,8 & 40,8 \\
\hline
\end{tabular}

Fuentes: Labour Force Statistics, OCDE / Estadisticas de Empleo OIT / Encuesta de Población Activa: INE / Tomado de Caja Laboral Popular, El desempleo en el País Vasco.

»El empleo en la agricultura es quizás el sector que ocupa una posición más cercana a la media europea, a la que además se aproxima de forma progresiva. Los ritmos de decrecimiento del empleo agrario han sido fuertes en el período considerado para el caso vasco, de forma que en 1978 sa 
población activa ocupaba tan sólo a un $9 \%$ del total, situación superada de entre los países considerados por Francia, Austria, Italia y el Estado español.» ${ }^{7}$

Es fácil suponer que las transformaciones en la estructura productiva, germen de las transacciones sectoriales de la PA ocupada, han de conllevar profundos cambios también en muchas otras órbitas del sistema social: imposición de un reciclaje profesional, pérdida del status socio-econórnico al pertenecer a un estrato profesional obsoleto, surgimiento de nuevas élites socio-profesionales de la mano de los nuevos sectores emergentes y un largo etcétera.

Todos los cambios apuntados y otros tantos derivados de aquétlos pue. den ser objeto de un estudio más profundo. Esto, aceptando las pautas de desartollo que imperan en el conjunto de países de la Europa avanzada, cuyos pasos no han de ser necesariamente ni los mejores, ni los únicos, y cuyas alternativas también son estudiables. De ahí que también sea preciso recordar el insuficiente conocimiento de los sistemas de valores que acom. pañan hoy día en el País Vasco a tales procesos económicos, sus detractores, sus defensores y sus posibles evoluciones.

\section{EL DESEMPLEO}

«Tal como está tecogido en la encuesta de población activa, son parados las personas que no están ocupadas $y$, aunque esto parezca una tedundancia, resulta de gran interés para interpretar las cifras de paro. En efecto, según esta definición el número de parados depende de la evolución de dos variables: la población activa y la población activa ocupada, y cualquier variación que se produzca en éstas incide en la marcha del desempleo. En este sentido, hay que recordar que la evolución de la población activa ha venido aliviando, de forma artificial, tensiones en el metcado de trabajo, haciendo que, desde el punto de vista estadístico, el número de parados sea inferior al que correspondería a una situación en la que la oferta no estuviera tan retraída.» ${ }^{8}$

Dicho con otras palabtas, que si muy amplio parece ser el sector de población que padece la situación de desempleo, en realidad aún es mayor,

7. Caja Laboral Popular, El Desempleo..., pp. 80-81.

8. Caja Laborai Populax, Economia Vasca. Informe 1982. 


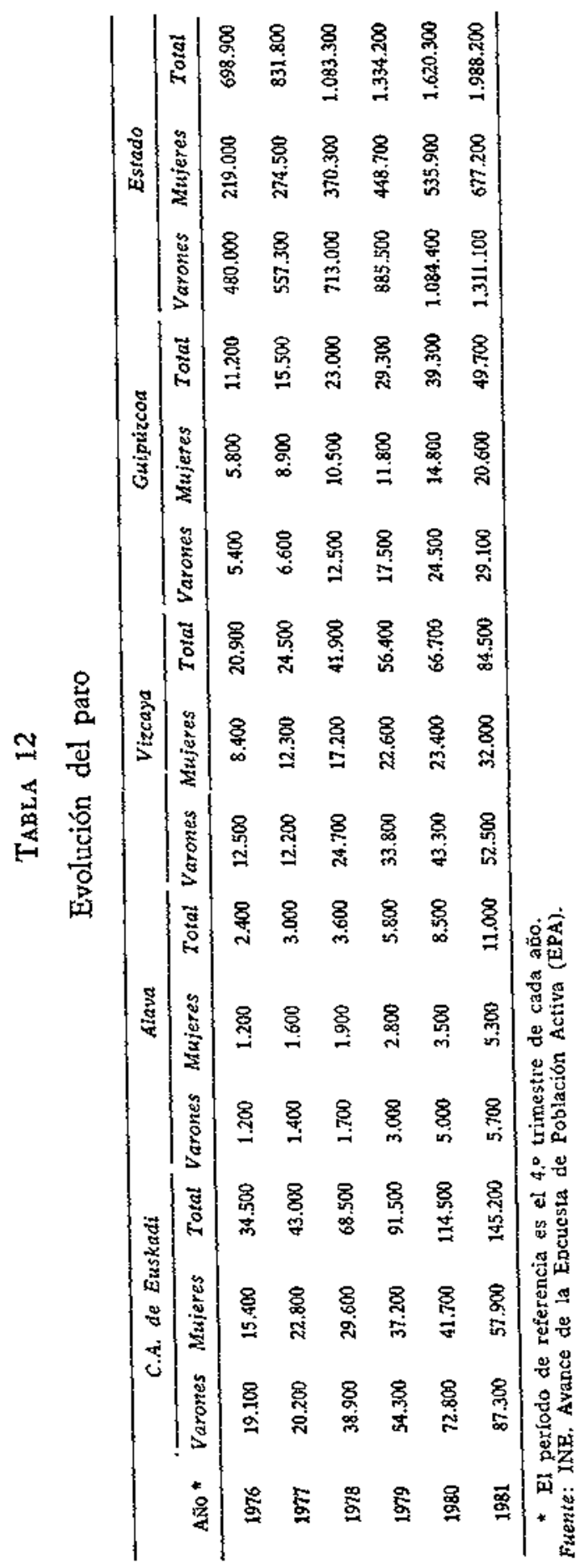


Mercado de trabajo y fuerzas sociales en Euskai Hetria Sur GRÁfICO 2

Evolución de la tasa de paro *

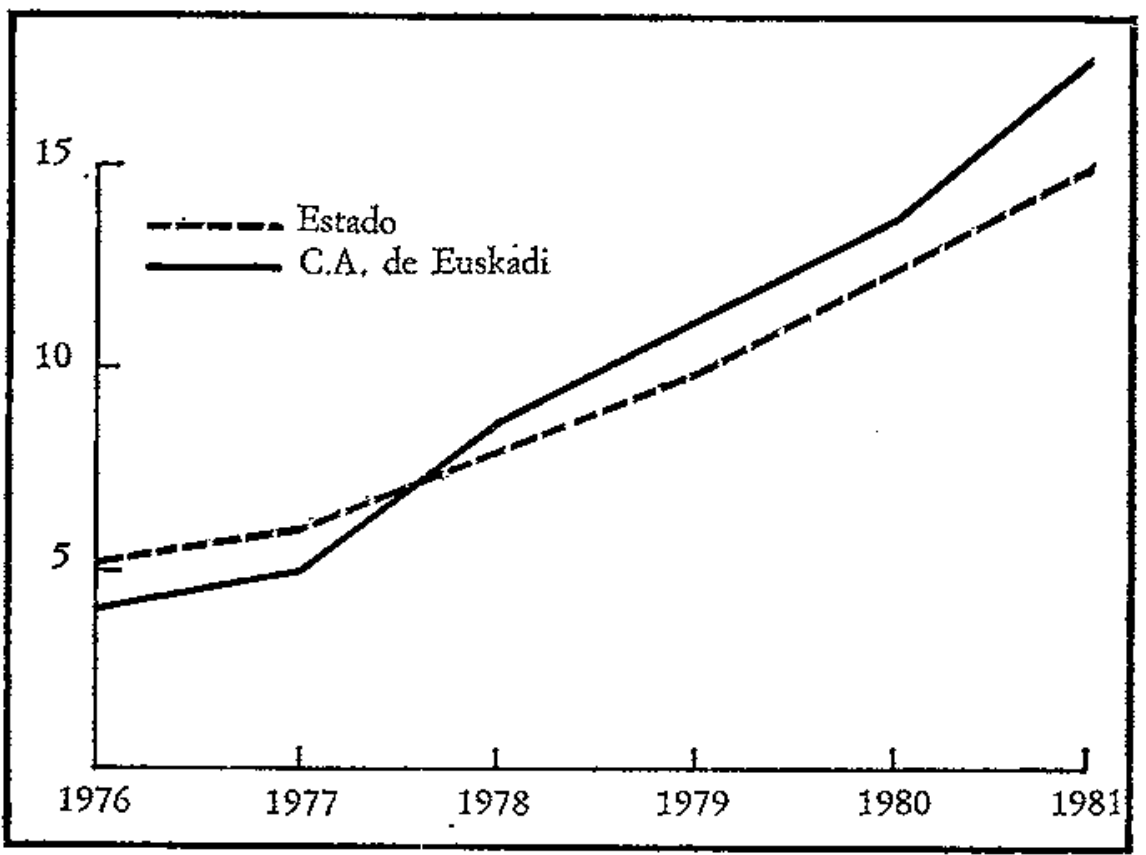

- Parados $\times$ 10:: población activa.

Fuente: Tomado de: Gobierno Vasco. Direccíó de Estadística. Anuario Vasco 1982.

ya que con las escasas perspectivas que existen de lograr un empleo, son muchos los que ni tan siquiera intentan entrar en el mercado de trabajo.

Este fenómeno del paro está entre los temas de mayor actualidad en el País Vasco, y por ello es de sobra conocido por todos. Concretemos aquí este saber colectivo sirviéndonos de algunas cifras (tabla 12). En 1976 las cifras de pato alcanzaban a un total de 34.500 personas en la Comunidad Autónoma Vasca; 5 años más tarde, la cifta total era de 145.200 , es decir, más de cuatro veces la ciftra de 1976. El gráfico 1 es suficientemente ilustrativo de este hecho, y también de la diferencia de in. tensidad con que esto mismo sucede en todo el Estado: en 1981 se da algo más del triple del pato existente en 1976 (1.988.200 y 698.900 parados, respectivamente). Vemos, pues, que la Comunidad Autónoma Vasca, aun partiendo de valores relativos inferiores a los existentes en el Estado en 
1976, en 1981 se encuentra con mayores cifras porcentuales que éste. Si las cifras en que nos hemos apoyado no incluian a Navarra, no quiere ello decir que allí el problema sea menor (se ha omitido hasta ahora por no fgurar en las fuentes utilizadas). Tenemos, por ejemplo, que en 1980 las tasas de paro eran las siguientes:

\begin{tabular}{lrr}
\hline Alava $\ldots \ldots \ldots \ldots \ldots \ldots \ldots$ & $8,5 \%$ \\
Guipúzcoa & $\ldots \ldots \ldots \ldots \ldots \ldots$ & $14,7 \%$ \\
Navarra $\ldots \ldots \ldots \ldots \ldots \ldots \ldots$. & $12,8 \%$ \\
Vizcaya & $\ldots \ldots \ldots \ldots \ldots \ldots \ldots$ & $15,1 \%$ \\
País Vasco Sur $\ldots \ldots \ldots \ldots$. & $13,9 \%$ \\
Estado & $\ldots \ldots \ldots \ldots \ldots \ldots$ & $12,6 \%$ \\
\hline
\end{tabular}

Navarra estaba por encima de la media estatal y más próxima a la provincia de tasa más alta, Vizcaya, que a la de tasa menor, Alava. En 1982, las cifras no son más optimistas y las tasas se reparten del siguiente modo: ${ }^{10}$

\begin{tabular}{|c|c|}
\hline $\begin{array}{l}\text { Álava } \ldots \ldots \ldots \ldots \ldots \ldots \ldots \\
\text { Guipúzcoa } \\
\text { Vizcaya } \ldots \ldots \ldots \ldots \ldots \ldots \ldots \ldots \ldots \ldots\end{array}$ & $\begin{array}{l}14,9 \% \\
18,8 \% \\
21,4 \%\end{array}$ \\
\hline Comunidad Autónoma ... & $19,7 \%$ \\
\hline Navarra ..... & $15,0 \%$ \\
\hline País Vasco Sur & $18,9 \%$ \\
\hline Estado ... & $17,1 \%$ \\
\hline
\end{tabular}

Alava sigue siendo la provincia que menor tasa de paro registra $(14,9 \%)$ y muy de cerca le sigue Navarra $(15,0 \%)$. Vizcaya es la que más padece esta situación con un $21,4 \%$, seguida de Guipúzcoa $(18,8 \%)$.

El conjunto de Euskadi Sur ha aumentado en 5 puntos la tasa de desempleo entre 1980 y 1982; en este mismo período, en el Estado se observa un incremento muy similar, 4,5 puntos. Por tanto, la intensidad del incremento sigue siendo mayor en Euskal Herria Sur, pero no mucho más

9. Caja Laboral Popular, El desempleo...

10. Caja Laboral Popular, ... Informe 1982. 
que en el Estado. En el caso del País Vasco, tras este creciente proceso de desempleo, podemos encontrar razones económicas: graves deficiencias estructurales en la industria ya apuntadas al comienzo de la crisis por algunos autores; ${ }^{\text {il }}$ otto aspecto de índole económica, evidentemente, sería la contracción de la economía mundial.

En el orden tecnológico podríamos apuntar: la obsoletización de gran parte de las instalaciones industriales, procedentes algunas de los tiempos de la Revolución Industrial (sobre todo en el caso de Vizcaya), y la pérdida de competitividad que ello ha ocasionado. Ligado a esto, tenemos la reconversión industrial que se ha acometido, y que en muchos casos implica una mayor automatización de los puestos de trabajo, con la consiguiente reducción de plantilla e inctemento del paro, Las consecuencias derivadas de esta situación son de dominio público en sus aspectos humanos y familiares. Su otra dimensión social, es decir, en su capacidad para provocat a medio y largo plazo grandes cambios en la sociedad no ha sido estudiada aún.

\section{Características del Desempleo}

Hernos visto anteriormente el incesante aumento del desempleo, algo más acuciado en el País Vasco Sur que en el conjunto estatal. Si atendemos a la evolución por sexo (tabla 13), vemos que para Euskal Herria Sur el porcentaje de parados se ha incrementado en 8,1 puntos desde 1980 hasta 1982, en lo concerniente a las mujeres. Para los varones el incremento ha sido de 3,5 puntos en el mismo petíodo. Al hablat de la evolución de la PA decíamos que la mujer se estaba incorporando progresivamente al mercado de trabajo, y ese mismo fenómeno se nos vuelve a aparecer ahora: muchas de las mujetes tecién incorporadas no encuentran empleo. ¿Lleva la mujer el camino de equipararse socialmente con el hombre? ¿Su salida de la marginación profesional en que se encuentra le arroja a otra nueva marginación: el paro? Si analizamos el desempleo por tamas de actividad, tenemos que: "La mitad de los parados pertenecen al grupo de no clasifi cables, lo que quiere decir que en su mayor parte son personas que no han tenido una ocupación anterior. Por otra parte, lo más destacable resulta la elevada tasa de paro $(29,6 \%)$ del sector de la construcción. ${ }^{12}$

11. Caja Laboral Popular, Pais Vasco. Estructura Industrial 1970-1975, ed. Lea poldo Zugaza, Durango, 1976.

12. Caja Laboral Popular, ... Informe 1982, p. 548. 
«Papers»: Revista de Sociologia

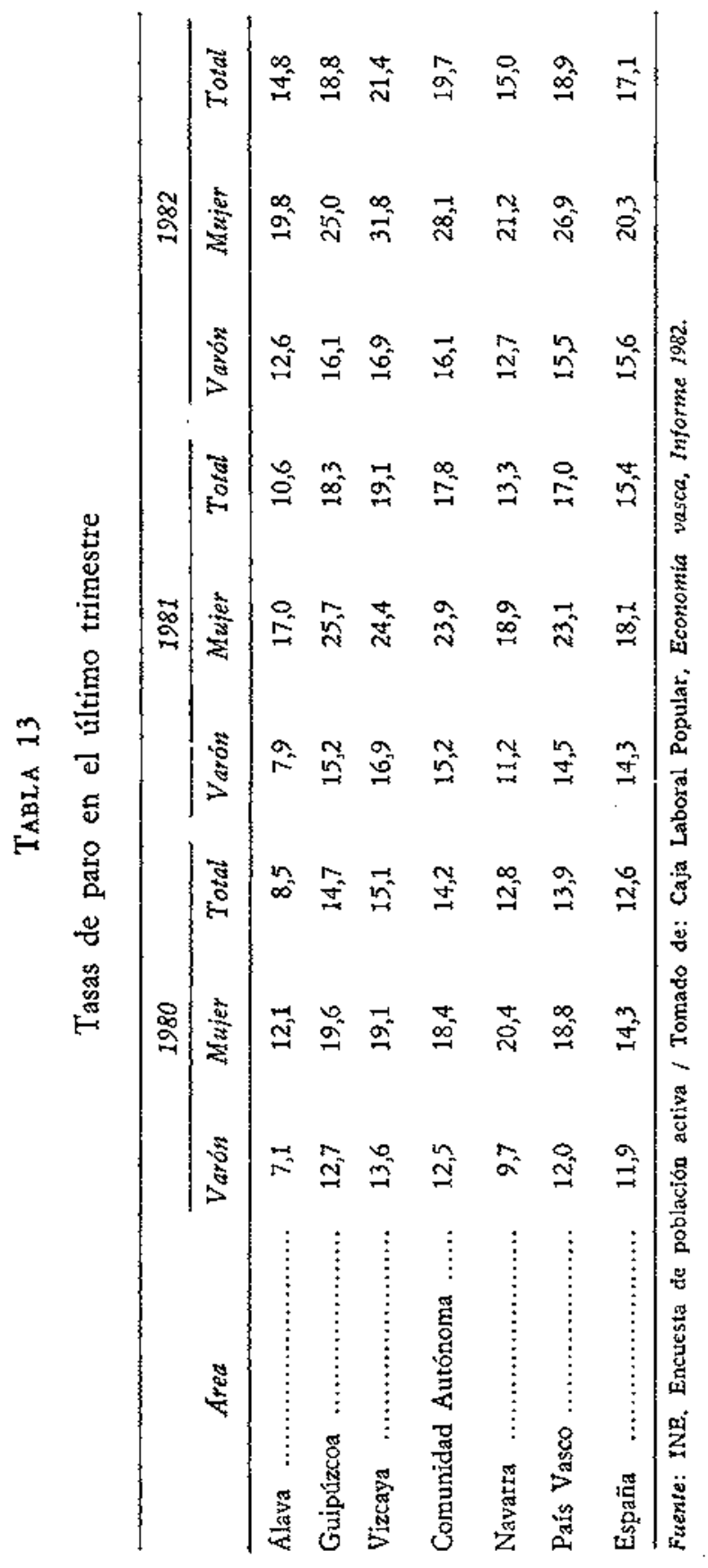


Si recordamos que las corrientes migratorias habian tenido un punto de inflexión, pasando de ser el País Vasco Sur tierra de inmigrados en las últimas décadas, a convertitse en foco de emigración en los momentos actuales, podremos fácilmente comprender que el sector de la construcción se halle sumido en profunda crisis y tenga aitas ciftas de pato: la demanda de viviendas se ha reducido considerablemente. Por otra parte, el creciente paro femenino pos la reciente incorporación de la mujer al mercado de trabajo, puede aparecernos incluido también al estudiar la edad, puesto que las mujeres que protagonizan tal cambio serán preferentemente jóvenes.

Deteniéndonos en la edad, tenemos que: «Mucho más clata aún es la situación vista desde la óptica de la edad. En el País Vasco Sur el 26,8\% de los parados tienen menos de 19 años y el $59 \%$ menos de 24 . Estas cifras ponen de manifiesto la gravedad del paro juvenil, que se puede apreciar mejox aún si se tiene en cuenta que el $70,6 \%$ de los jóvenes vascos menores de 19 años están sin empleo y que se encuentran en la misma situación el $51,6 \%$ de los menores de 24 años. $)^{13}$

Evidentemente, las crecientes cifras de desempleo de los distintos colectivos sociales (jóvenes y adultos, hombres y mujeres, técnicos y trabajadores manuales, etc.) han de conllevar profundas transformaciones sociales. Pueden darse cambios decisivos en: las expectativas de vida (piénsese en los jóvenes, por ejempio); en los compottamientos sociales: anomía (en el sentido de carencia de medios adecuados para la obtención de unos fines) y sus posibles detivaciones delictivas; en los sistemas de valores sociales (tecordemos el todavía vigente valor supremo dei trabajo); en el nivel de renta de la población, etc.

Como consecuencia de esto, las fuetzas sociales que defienden ideolo gías, creencias e inteteses, de cualquier signo que sean, habrán de modificar sus estrategias y tácticas si quieren dar respuesta a los tiempos actuales. Al hablar del paro, nos viene en seguida la imagen de los sindicatos. En el caso de los mayoritarios, es conocida la dejación que del problema de los parados se viene haciendo. Es un hecho, que de unos planteamientos más radicales y reivindicativos que postulaban hasta la anterior década, han pasado a otros más consensuados (con el capital), aceptando incluso un descenso en la capacidad adquisitiva de los trabajadotes. Peto del tema de los parados, bien poco o nada. Otras fuerzas tanto sindicales como políticas de la extrema izquierda han comenzado a defender nuevos programas que implican un radical cambio en los valores sociales vigentes: «Trabajar menos, para trabajar todos.»

13. Caja Laboral Popular, op. cit., p. 548. 


\section{CONCLUSTONES}

Se han omitido muchos aspectos importantes para un estudio del «mercado de trabajo» y de las «fuetzas sociales», algunos comunes a cualquiet área geográfica y realidad social actual (evolución y perspectivas de la economía internacional, paro real y paro registrado, conflictividad laboral, etc.), y otras más específicas del caso vasco (viabilidad y orientación de la recon. versión industrial, valoración del euskera en el mercado de trabajo, etc.). Se es consciente de tales omisiones.

Por otro lado, se ha insinuado repetidamente que Euskal Herria Sur está en un momento crítico, el más crítico desde la guerta del 36 . Su situación económica dista mucho de ser buena, aunque los últimos informes relativos a 1982 parecen apuntar una ralentización en la caída de la economía.

Pero a esto hemos de sumat la catástrofe acaecida el pasado agosto. ¿Puede haber sido el golpe definitivo para una caída económica sin fondo? ¿Se dará en consecuencia una grave crisis social de insospechadas consecuencias? ¿Habrán sido las inundaciones el f́ltimo capítulo de una dura época, a la que seguirán otros tiempos mejores? ¿Qué mođelo de sociedad resultaría de ello? Como puede verse, el momento tiene un tremendo atractivo para que los investigadores sociales nos lancemos a su estudio. 


\section{BIBLIOGRAFIA}

Caja Laboral Populat

- País Vasco. Estructura industrial 1970-1975, eđ. Leopoldo Zugaza, Durango, 1976.

- El desempleo en el País Vasco, 1982.

- Economía Vasca. Informe 1981, 1982.

- Economía Vasca. Informe 1982, 1983.

Cárnaras de Cometcio Vascas

- Boletin de coyuntura y estadistica del Pats Vasco.

Gobierno Vasco. Dirección de Estadística

- Evolución de la población. Pevíodo 1900-1981, 1982.

- Censo de población y vivienda 1981 de la Comunidad Autónoma de Euskadi. Estructura de la Poblacion, 1983.

- Anuario estadístico vasco 1982, 1983.

Instituto Nacional de Estadística

- Encuesta de Población Activa.

Llera Ramo, Francisco José

- La estructura social del Pais Vasco, «Rev. Internacional de Sociología», separata, Madrid, octubre-diciembre 1982.

Ministerio de Economía y Cometcio

- Análisis de las estadísticas del mercado del trabajo, 1982. 